\title{
CORRECTION
}

\section{Correction to: Cross-modal commutativity of magnitude productions}

\author{
Wolfgang Ellermeier ${ }^{1}\left[\right.$ Florian $_{\text {Kattner }}^{1}\left[\right.$ [D $\cdot$ Anika Raum $^{1}$
}

Published online: 15 October 2021

(c) The Author(s) 2021

\section{Correction to: Attention, Perception, \& Psychophysics (2021) 83:2955-2967 https://doi.org/10.3758/s13414-021-02324-y}

The following errors in the published article need correction:

Column 4 of Table 2 (Appendix): Bayes factors erroneously computed for independent observations. Table 2 with the corrected values appears below.

Consequently, the statement summarizing the Bayesian statistics on commutativity in the results section needs to be changed (p. 7 of the pdf): Rather than "in eight instances", it should be "in eighteen instances" based on the corrected computation of Bayes factors.

Publisher's note Springer Nature remains neutral with regard to jurisdictional claims in published maps and institutional affiliations.

The original article can be found online at https://doi.org/10.3758/ s13414-021-02324-y

Wolfgang Ellermeier

ellermeier@psychologie.tu-darmstadt.de

1 Institute of Psychology, Technical University of Darmstadt, 64283 Darmstadt, Germany 
Table 2 Wilcoxon signed-ranks tests indicating violations of cross-modal commutativity and corresponding Bayes factors in favor of the null hypothesis of the axiom to hold $\left(\mathrm{BF}_{01}\right)$

\begin{tabular}{|c|c|c|c|c|c|c|}
\hline \multirow[b]{3}{*}{ Subject } & \multicolumn{2}{|c|}{ Wilcoxon test } & \multirow[t]{2}{*}{ Bayes factor } & \multirow{2}{*}{\multicolumn{2}{|c|}{$\frac{\text { Wilcoxon test }}{X^{l b l}(l \rightarrow b \rightarrow l)}$}} & \multirow[t]{2}{*}{ Bayes factor } \\
\hline & \multicolumn{2}{|c|}{$X^{b l b}(b \rightarrow l \rightarrow b)$} & & & & \\
\hline & $z(V)$ & $p$ & $B F_{01}$ & $z(V)$ & $p$ & $B F_{01}$ \\
\hline HOED & -1.88 & .060 & 0.797 & -0.83 & .409 & 2.776 \\
\hline MINI23 & 0.69 & .490 & 4.600 & -1.80 & .094 & 0.978 \\
\hline KLCH06 & -1.57 & .116 & 1.240 & 0 & 1 & 4.977 \\
\hline VOSO31 & 0.06 & .950 & 4.982 & -1.14 & .256 & 2.540 \\
\hline HEKA29 & 0.85 & .396 & 3.645 & -1.37 & .166 & 1.397 \\
\hline DIAN07 & -1.76 & .079 & 1.556 & -2.07 & .039 & 1.114 \\
\hline ZESI15 & -0.38 & .715 & 4.475 & -0.91 & .362 & 3.420 \\
\hline SESV24 & -1.26 & .208 & 1.446 & -1.58 & .115 & 1.129 \\
\hline IGMA08 & -2.32 & .020 & 0.393 & 0 & 1 & 4.575 \\
\hline HAKA24 & -0.66 & .509 & 3.776 & -0.69 & .489 & 3.829 \\
\hline HEAN19 & -1.00 & .326 & 2.665 & -0.77 & .441 & 3.032 \\
\hline FRBR05 & -2.07 & .035 & 0.390 & -0.50 & .615 & 3.511 \\
\hline ECGE12 & 0.13 & .900 & 4.621 & -1.30 & .195 & 1.757 \\
\hline KAGA10 & -0.63 & .530 & 4.857 & 1.57 & .116 & 1.905 \\
\hline RUMA10 & -1.13 & .258 & 4.301 & -1.47 & .141 & 1.563 \\
\hline SEIR22 & 1.61 & .108 & 0.534 & -1.30 & .195 & 2.033 \\
\hline JEGR05 & -0.31 & .753 & 4.038 & -1.07 & .283 & 3.252 \\
\hline STRE25 & -2.98 & .003 & 0.084 & -0.63 & .529 & 4.332 \\
\hline FRAL10 & 0 & 1 & 4.920 & -1.51 & .131 & 1.254 \\
\hline THMA12 & 1.57 & .119 & 1.169 & -1.04 & .298 & 2.151 \\
\hline
\end{tabular}

Note. z-scores for the Wilcoxon test statistic V and corresponding p-values are reported. All $p<0.1$ shown in bold type. Bayes factors indicate how likely the null hypothesis of the axiom to hold is, compared to the alternative hypothesis of a violation of commutativity 